Rev. Chil. Pediatr. 58(1): 47-49, 1987

\title{
Tratamiento de las micosis superficiales del niño con tolciclato
}

\author{
Dra. Acindea Jara P.1: bra, Y. Sotedad Quero B.2
}

Treatment of skin fungal infections with tolciclate

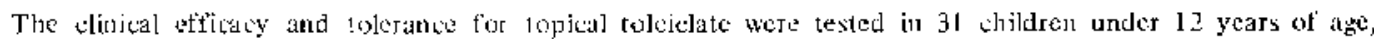
aftected by different kinds of lungal infection of $t$ le skin, incleding 19 patients with tinca citcinata (microspor um

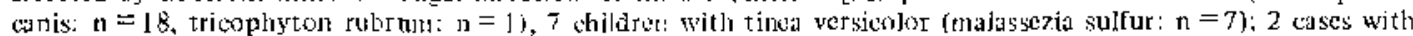

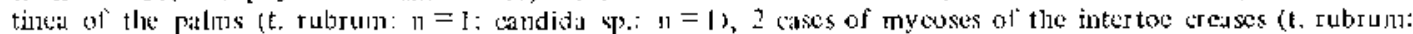

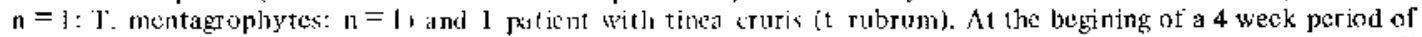
trcatment $74 \%$ ot the patient somplained of pruitus, there was evidence of sealing in $87 \%$ and of erithema in $74 \%$ of the cases. At the end of the study, signs and symptoms load disiapeated trom 29 (93.5\%) patients and fungus were isolated only from two cases (1. rubrum: $n=1$, and ni. fustur; $n=1$ )

(Key words: tolciclate, antifungal topical therapy, skin mycoses, lungal skin infections).

E] tolcielato (Tolmicen (E) es un nuevo derjvado tiocarbímico. cuya composición química corresponde at 0 (1, 2, 3.4-tetrabidro-1,4. metanonaftalen - $6-i 1)-m$. $N$ dimetiltiocarbanilato ${ }^{\perp}$. Su eficacia clinica ha sido demostrada en diferentes inas causadas por dermatofitos $y$ en pitiriasis versicolor ${ }^{2}, 3,4,5$. Su efecto "in vitro" parece superior al de otros fármacos antimicóticos y similar al tolnaftato. La mayor lipasolubilidad del tolciclato, podría explicar los mejores resultados obtenidos en clínjcat sobre el tolnafia. to ${ }^{6}$.

1. Servicio de dermatología, Hospital Luis Calvo Mackenna.

2. Departamento de Microbiología, División Ciencias Médicas Norte, Facultad de Medicina, Univcrsidad di Chïle.
Dilerentes trabajos clínicos, en su mayoria en pacientes adultos, demuestran que el medicamento es bien tolcrado atin en tratamientos prolongados $y$ que las principales dermatomicosis sensibles al fármaco son la tiña corporis y la tiña cruris. Utimamente se ha publicado efectos fayorables del tolciclato en candidiasis cutáneas ${ }^{7}$. Con respecto a su acción terapéutica y tolerancia en dermatomicosis del niño, existe escasa información extranjera ${ }^{8,9}$ razón por ta cual hemos estudiado sus efectos en enfermos menores de 12 años con algunas formas de micosis superficial.

\section{MATER IAL Y METODO}

Tolciclato fue craluado en 31 pacientes ambulatorios rue consultaron al servicio de dermatolog ta del Hospital "Luis Calvo Mackensa", on quienes se confirmó el 
diagnóstico de dermalcotnicosis mediante los sxámejus pertinentes. Su edat promedio lue 8,9 años íango 2 a l2 añosl y 17 (55\%) èran varones.

lodos los entermos fueron sometidos a un examen clinico completo intertogandose pxi filctures predjsponentes, tratamientos previos y encernedades concomitantes. Se deseartaron niños con tratamientos testeroidales o inmunusupresores. I.os casos habín recibido medicumentos antimicóticos oon anterioridad, lucron dejados por ult mínimo de un mes sin tratimicento anles de admitirtos al estudio.

La evaluación clínica tomó en consideración los siguientes sintonas y sipnos: eritema, desceimación, prur ito, vesículas, fïsuras y alteraciones del pelo. Con el fin de ponderar estos parámétros, ex dencosibió en cuda control, la exkensión de la lesión y los camlios experimentados durante el tratamiento, mediante unit escala arbitraria donde al cada signo se le fijo un valur: 0 (ausenten; 1 (leve); 2 (moderido) y 3 intenso). Low exámenes de laboratorio incluyeron estudios microscí picos de cseamas de las zonas atectedas usando $\mathrm{KOH}$ al $20 \%$ y cultivos para ]onjos on aear Saboutcaud grlucoSaব̆o adicionado de cloramtenicol $y$ agar Saboureaud adicionado de astidiona, por un período de 30 dias

Todos los pacientes fueron controlados semanalmerti hasta completar tas 4 semplas de tratamiento corl [o]ciciato, anotándose la cvolución de tos símomis y los eumbios experimentados por las lesiones. Al inicio y al términe del tratamianto se practicaron fos estudios micológicos, descartando a itepullos cufermos en quienes, en el nomento de la adruisión éstox dieron resultados nevativos.

1. I incdicamento fue usado en crema al $1 \%$ dos aplicilciones diarias, durante 30 dijas. Se instruyó a los padres para que no cubrietan las lesiones con vendas o apúsitos. ni dieraja otros medicartuentos sin previa autorinacion del medico tratante. Al termino del tratamiento sc realizós una evaluatión global de lat efijeacia del medicamento, para to cula se establecieron los sizuientes criterios: euración finvestigación negativa p.rma hongos, directo y cultivo; remisión totul de for sintoms $y$ signos): mejoría (Iesuldado del examen directo o cultivo positivos, con mejoria de los sintomas, o los sigros a ambosy: sin modifiăciones dfalla clinica y de laboratorio, sin armbios respecto al ingreso).

\section{RESULTADOS}

El número mayor de casos correspondía a tifia circinada $(\mathrm{n}=19 ; 62 \%)$ y pitiriasis versicolor $(n=7 ; 23 \%)$ y en menor número a tin̄a palma $(\mathrm{n}=2)$, thicosis interortejo $(\mathrm{n}=2)$ y tiña crural $(n=1)$.

Los patónenos aislados en los estudios micológicos (directos y cultivus) se desctiben en la Tabla 1: el microsporum canis fue el agence mas frecuentemente identificado en tas tinas circinadas ( 18 casos), excepto un caso producido por tricophyton rubrum.

En todos los pacientes portadores de pitiriasis versicolor se identificó, en los exámenes de escamas, malassezia furfur. El trycophyton rubrum fue responsable de 1 caso de tiña palmar, ot ro de tiña crural, de un caso de micosis interortejo además de uno de los pacientes descritos entre las tir̃as circinadas. La candida $s p$ fue identificada en
Tabla 1.

Resultados de los estudios micológioos en 31 niños portadores de micnsis cutánea

\begin{tabular}{lrr}
\hline Palogeno & 10 & $\%$ \\
\hline Microsponum canis & 18 & 58 \\
Malassezde furfur & 7 & 23 \\
Tricophyton rubrum & 4 & 13 \\
Tricophyton montagrophyes & 1 & 3 \\
Candida sp. & 1 & 3 \\
\hline
\end{tabular}

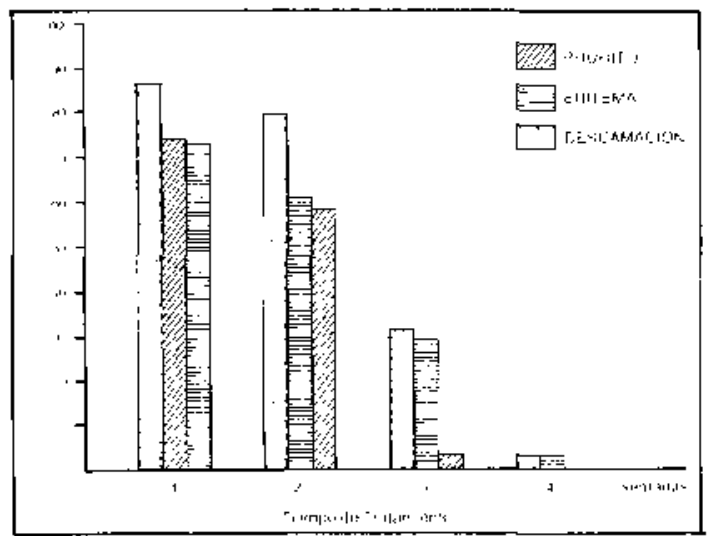

Figura 1. Porcentaje de pacientes asintomations in relación al tiempo de trititmiento ton Tolciclato.

un niju con 1 išì palmar. Finalmente, de otro enfermn, con micosis interortejos se cultivi un tricophyton mentagrophytes.

Fin el momento del ingreso habia eritema en $74 \%$ de los casos descamación en $87 \%$ y prurito en $74 \%$. La modificación de estos sintomas y signos se describe en la Fig. 1: el prurito desapa reció más rápidamente que los demás signos, y sólo en un paciente ( $3 \%$ ) persistía después de la segundit semilna de tratamiento. El eritema y la descumación mejoraron paralelamente, de tal forma que los 15 dias de evolucicin, labian $6 \mathrm{y}$ $80 \%$ de los enfermos con estos signos. Al término de la investigación, sólo en un enfermo persistía el eritema y en otro la descamación: uno con tiña palmar y el otro a pitiriasis wersicolor $(6,5 \%)$.

Las modificaciones experinctitadas por los sintomas y signos. entre las diversas yariedades de tiña, no mostraron diferencias significativas. En general. en los pacientes con pitiriasis versicolor la mejor ía fue más lenta. como era de esperar en este tipo de micosis, pero, salvo et uno, portador de un proceso extenso de tronco, cuello y extremidades, las manifestaciones habían desaparecido a la cuarta semana en la mayoría de los casos.

En los 7 pacientes con pitiriasis versicolor, los exámenes micológicos directos eran negativos a la segunda semana de tratamiento en $71 \%$ y, sólo en el mismo enfermo comentado anteriormente, 
persistían los resultados positivas al término del seguimiento. En los restantes pacientes con tifias cutáneas, los exámenes para hongos eran negatjvos a la cuarta semana de tratamiento, con la unica excepción de un niño portador de tiña palmar en cuyos cultivos persistía un tricophytont nobrum. En sintesis, al termino del tratamjento se aislaron hangos de 2 pacientes $(6,5 \%)$ solamente.

Una evaluación global de la efícacia de tratamiento demostró que todos los pacientes curaron o experimentaron franca mejoría de los síntomas $y$ signos físicus con que ingresaron al estudio.

No se detectaron reacciones alérgicas $u$ otros efectos colaterales atribuibles al medicamento.

\section{DISCUSION}

Las distintas afecciones causadas por hongos son motivos frecuentes de consulta en niños con enfermedades cutáneas. Se dispone de diversos preparados aparentemente eficaces, pero muchas veces un seguimiento adecuado demuestra lo contrario. Esta evaluación del tolciclato mostró una alta proporción $(93.5 \%)$ de curaciones en nin̄os con dermatomicosis, con desaparición de los hongos de los cultivos y exámenes directos y la desaparición đe los sintomas y signos $(93,5 \%)$ al término de cuatro semanas de tratamiento. Si bien la mejoria de los pacientes con pitiriasis fue más lenta, ocurrió en una proporción tambièn bastante alta. Estos resultados son muy parecidos a los obtenidos por atros autores ${ }^{8}$.

Raggio y col. ${ }^{10}$ compararon, con método de doble ciego, los efectos de tolciclato y tolnaftato en pacientes con pitiriasis versicolur, mostrando 92,5\% de exámenes micológicos negativos después de 2 semanas de tratamiento en el primer caso, versus $64 \%$ en el segundo.

Los estudios "jn vitro" no han confirmado que tolciclato sea activo sobre las candidas sp., sin embargo en nuestro único caso y en otra investigación, doble ciego, los enfermos mejora. ron después de la cuarta semana de tratamiento con el medicamento ${ }^{7}$. Una explicación a esta aparente discrepancia es que la candida, en un ambiente pobre de nutrienies como la piel, adoptaría formas miscelares que pueden ser destruidas por el medicamento, a diferencia de las esporuladas que tienden a ser resistentes.
Este estudio sustenta la eficacia terapéutica del tolciclato, y su buena tolerancia por parte de niños. con diferentes formas de dermatomicosis

\section{RESUMEN}

Mediante un estudio abierto, se estudió la eficacia clínica y la tolerancia para tolciclato, en 31 niños con diferentes dematomicosis, todos menores de 12 at̃os de edad. Las principales dermatomicosis fueron tiñas circinadas $(n=19)$, tiña cruris $(n=1)$, tiřa palmar $(n=2)$, pitiriasis versicolor $(n=7)$ y micosis interortejo $(n=2)$. En $90 \%$ de los casos se demostró desaparición de la evidencia de infección en los exámenes direc. tos y cultivos para hongos y en $94 \%$ desaparición de las manifestaciones clinicas. No se observaron sintomas ni signos de reacciones adversas en esta serie.

\section{REFERENCIAS}

1. De Corneri, I. et col.: Tolciclate against dermatophytes. Arzneimittelforsch 26: 769, 1976.

2. Intini, C, Batlaglia. A., Mangiarotti, A.M., Picco, $A . M$. . Viaro, $D$. and Sacchetti. G.: Multicentre clinical study with Tolciclate in the local treatment of skin mycoses in 1083 patients. Pharmathera. peutica 2: 439,1980 .

3. Ryley, $J$ : Experimental approaches to antifungal chemotherapy. Advances in phasmacology and chemotherapy, 88: pp. 49-176.

4. Grixoni, F., Lorenzoni, A., Muzzol, L.: Sperimentazionc d'un nuovo preparato antimicotico per uso topico il Tolciclato. S. Mal. Infett. 31: 165, 1979.

5. Melloni, P., Metelli, R., Vecchietri, V., Logemann V., Castellino, S., Monti, $G$., De Cameri, I.: New antifungal agents. Eur. J. Med. Chem. 9: 26, 1974

6. Bianchi, A., Monti, G., De Carneri, I.: Tolciclate: Further antimicotic studies. Antimicrob Agents Chemother, 12:429, 1977.

7. Battaglia, A., Ittini, C., Perbellini, A., Guglielminetti, M., Viaro, D., Sacchetti, G.: Tolcichte in the local treatment of skin candidiases: a double blind cantrolled multiclinic trial versus Clotrimazole. J. Int. Med. Res. 10: 367, 1982.

8. Henrique, C.L.: Tolciclato no tratamento das micoses superficiais da infancia e adolescencia. An. Bras. Dermatol. 58: 183, 1985.

9. Jacohs, $P$.: Fungal infections in Childhood. Pediutr. Clin. North Am. 25: 357, 1938

10. Raggio, $X$.: Estudio comparativo de la actividad de un nuevo antimicótico, Tolciclato, en pitiriasis versicolor. Bol. Mic. 2: 5, 1984. 